Questions vives

\section{Questions Vives}

Recherches en éducation

Vol. $5 n^{\circ} 11 \mid 2009$

Le développement professionnel : quels indicateurs?

\title{
Les indicateurs temporels du développement professionnel des professeurs des écoles néo- titulaires : entre temporalité institutionnelle et temporalité personnelle?
}

\section{Alexia Stumpf et Michel Sonntag}

\section{(2) OpenEdition}

\section{Journals}

Édition électronique

URL : http://journals.openedition.org/questionsvives/574

DOI : 10.4000/questionsvives.574

ISBN : 978-2-8218-1082-2

ISSN : 1775-433X

Éditeur

Université Aix-Marseille (AMU)

\section{Édition imprimée}

Date de publication : 1 janvier 2009

Pagination : 177-191

ISBN : 978-2-912643-35-3

ISSN : 1635-4079

\section{Référence électronique}

Alexia Stumpf et Michel Sonntag, «Les indicateurs temporels du développement professionnel des professeurs des écoles néo-titulaires : entre temporalité institutionnelle et temporalité personnelle?», Questions Vives [En ligne], Vol.5 n¹1 | 2009, mis en ligne le 01 juin 2011, consulté le 10 décembre 2020. URL : http://journals.openedition.org/questionsvives/574 ; DOI : https://doi.org/10.4000/ questionsvives.574

Questions Vives est mis à disposition selon les termes de la licence Creative Commons Attribution Pas d'Utilisation Commerciale - Pas de Modification 4.0 International. 


\title{
Les indicateurs temporels du développement professionnel des professeurs des écoles néo-titulaires : entre temporalité institutionnelle et temporalité personnelle?
}

\author{
Alexia Stumpf 1 , Michel Sonntag²
}

Résumé: Notre recherche explore la question du développement professionnel des professeurs des écoles néo-titulaires, c'est-à-dire des enseignants débutant dans le métier et qui comptabilisent entre une et trois années d'expérience sur le terrain, après leur sortie d'IUFM. II apparaît que ce long continuum, qu'est le processus de développement professionnel enseignant, est caractérisé par des phases. Celles-ci s'inscrivent dans une double temporalité à la fois institutionnelle et personnelle. Notre article vise à mettre au jour les différentes phases telles qu'elles sont énoncées dans les discours officiels et à travers les entretiens que nous avons menés avec des professeurs des écoles néo-titulaires. II repose donc sur une analyse conjointe de textes officiels et d'entretiens. In fine, nous interrogerons la manière dont les discours institutionnels, sur l'entrée dans le métier, font ou ne font pas sens pour ces professeurs des écoles néo-titulaires.

Mots-clés : Développement professionnel, professeurs des écoles, néo-titulaires, rapport au temps

Abstract: Our study explores the question of the professional development of new primary school teachers, meaning teachers who are starting in their teaching career and who have accumulated between one and three years experience in the classroom after they have finished their training in the "IUFM". It seems that this long continuum that is the process of professional development is characterized by phases. These are both institutional and personal. Our article aims to shine light on the different phases as they are demonstrated in the official literature and through the research that we have carried out talking to new teachers. This study therefore, rests on evidence based both on official literature and one on one interviews. In short, we are questioning the way that official literature does or does not make sense for post graduate school teachers, on the subject of entry in the teaching career.

Keywords: professional development, primary school teachers, new teachers, relation between teachers and time

\footnotetext{
1 ATER sciences de l'éducation à L'Université de Strasbourg Laboratoire: LISEC (Laboratoire Interuniversitaire des Sciences de l'Education et de la Communication), EA 2310.

2 Professeur en sciences de l'éducation à l'INSA de Strasbourg - Laboratoire : LISEC (Laboratoire Interuniversitaire des Sciences de l'Education et de la Communication), EA 2310.
} 

écoles néo-titulaires : entre temporalité institutionnelle et temporalité personnelle

Depuis près d'une décennie, le développement professionnel des enseignants fait l'objet de nombreuses recherches scientifiques (Borko, 2004 ; Clement, Vandenberghe, 1999 ; Day, 1999 ; Elmore, 2002 ; Garet et al., 2001 ; Guskey, 2002). Si les recherches qui prennent pour objet cette notion sont majoritairement anglo-saxonnes ou québécoises, cette question est aussi centrale dans les travaux menés par des chercheurs français, comme l'équipe du Crefi (Centre de Recherches en Education, Formation et Insertion) de l'Université de Toulouse ou dans des recherches sur les nouvelles pratiques professionnelles (Marcel 2005, 2007).

Toutefois, le caractère un peu polysémique de cette notion, pour l'heure non stabilisée, se traduit, dans le contexte francophone, par l'emploi d'expressions plus ou moins synonymes comme : formation continue (Boucher et L'Hostie, 1997 ; Lafortune, Deaudelin, Doudin et Martin, 2001; Ministère de l'Education Nationale français), perfectionnement, développement pédagogique (Lafortune et al., 2001), développement de carrière, évolution professionnelle (Huberman, 1989; Huberman, Grounauer et Marti, 1989), apprentissage continu (Gouvernement du Québec, 2001), croissance professionnelle (Glatthorn, 1995; Kagan, 1992). " On voit déjà, à ce niveau, la confusion entre le sens du concept lui-même et les moyens par lesquels le développement professionnel se réalise" (Mukamurera, Uwamariya, 2005).

A l'intérieur de cette problématique, l'idée de continuum semble constituer un point de convergence entre les écrits ministériels français et québécois notamment, au-delà de la diversité des expressions qui s'apparentent à la notion de développement professionnel. Celui-ci reposerait donc sur un apprentissage du métier qui se tisserait tout au long de la carrière, depuis la formation initiale.

Ce continuum est aussi caractérisé par des étapes qui jalonnent la construction progressive du développement professionnel enseignant. II s'inscrit dans une double temporalité à la fois institutionnelle et individuelle. Comment les discours officiels énoncent-ils ces étapes ? Comment les enseignants en parlent-ils ? Quelles transformations sont censées accompagner le processus de développement professionnel ? Qu'en disent les enseignants concernés? Quels sont les outils dont dispose l'institution pour procéder à l'évaluation du développement professionnel des personnels ?

Le présent article vise à identifier et interroger les étapes du développement professionnel des professeurs des écoles néo-titulaires, telles qu'elles sont énoncées dans les discours officiels et telles qu'elles sont parlées par les enseignants. Les étapes institutionnelles et les étapes personnelles s'inscrivent-elles dans la même temporalité ? Le découpage institutionnel du continuum fait-il sens, du point de vue des enquêtés ? Nous nous intéresserons plus particulièrement aux trois premières années d'exercice, qui constituent l'entrée dans le métier.

Pour ce faire, après avoir défini la notion de développement professionnel, nous procèderons, dans un premier temps, à une analyse de contenu d'un corpus de textes officiels pour identifier les étapes dudit développement, prévues par l'institution, dans la 
Le développement professionnel : quels indicateurs?

formation des enseignants. Celles-ci constitueront les indicateurs (temporels) institutionnels du développement professionnel.

Dans un second temps, nous chercherons à mettre au jour les étapes du développement professionnel, telles qu'elles sont perçues et vécues par des professeurs des écoles néotitulaires, à partir des propos tenus sur leur formation initiale et leur entrée dans le métier. Dans ce dernier cas, nous avons procédé à une analyse de contenu d'un corpus constitué de trente entretiens semi-directifs afin d'identifier les marqueurs temporels présents dans les discours et associés aux sentiments de changements, et notamment de progrès et d'autoefficacité, exprimés. Plus précisément, nous avons relevé, de manière systématique, les indicateurs que constituent les embrayeurs temporels, associés à la verbalisation d'un sentiment global de performance et de changement, afin de mettre au jour, in fine, le rapport au temps dans lequel s'inscrivent et évoluent professionnellement les néo-titulaires.

\section{La notion de développement professionnel \\ De l'approche condorcetienne de la notion de développement professionnel...}

Comment expliquer l'intérêt des chercheurs en éducation pour la notion de développement professionnel, si ce n'est à la lumière de l'évolution parallèle du monde du travail ? En effet, les « travailleurs du savoir » (dont les enseignants) participent de manière croissante, depuis deux décennies, à la réussite économique des pays développés, entrés dans l'ère de l'économie de la connaissance. Cette fonction permet de comprendre et de légitimer l'intérêt croissant des gouvernements pour l'éducation et la formation, ferments du capital humain, qu'il va falloir faire fructifier, dans un souci d'optimisation, tout au long de la vie. Ces politiques éducatives s'inscrivent dans le sillon de la philosophie condorcetienne puisque son auteur est considéré comme le précurseur de l'éducation permanente, l'instruction devant "emballer tous les âges" (Condorcet, 1792). Celle-ci repose sur le principe de perfectibilité de l'esprit humain. Ainsi, cette philosophie, clef de voûte des sociétés apprenantes, est devenue centrale également dans la formation des enseignants puisqu'elle va leur permettre l'actualisation des compétences, tout au long de leur carrière. Elle s'est traduite outre-Manche et outre-Atlantique par l'expression « lifelong learning ».

\footnotetext{
...à l'approche axée sur la professionnalisation

Les travaux qui prennent pour objet la notion polysémique de développement professionnel, chez les enseignants, s'inscrivent dans deux courants théoriques émergeant au-delà de la diversité des écrits recensés: l'approche développementale et l'approche professionnalisante (Mukamurera, Uwamariya, 2005). Nous ne développerons pas ces deux approches de manière extensive puisque tel est l'objet de l'article cité supra mais il nous importe néanmoins de souligner que, dans une approche développementale, le développement professionnel est pensé dans « une vision chronologique et linéaire. Or les événements survenus depuis les années 1960 (crises économiques, réajustements structurels, etc.) ont contribué à modifier l'image des professions et des parcours des individus (Dolan et al., 1995 ; Huberman et al., 1989). Dans ce nouveau contexte, penser à la stabilité de l'emploi et à une évolution professionnelle à caractère linéaire devient pratiquement impossible " (Mukamurera, Uwamariya, 2005). Aussi, l'approche professionnalisante constituera-t-elle notre ancrage théorique. Quelles en sont les caractéristiques?
} 
Dans cette approche, le développement professionnel est « vu comme un processus d'apprentissage. En effet, l'enseignant est considéré comme un apprenant qui, au fil du temps, construit et reconstruit ses savoirs. La vision de l'apprentissage est ici constructiviste dans la mesure où les expériences pratiques personnelles sont à la base du développement de savoirs. De plus, le processus d'apprentissage est à la fois individuel et collectif » (Mukamurera, Uwamariya, 2005). Plusieurs modèles coexistent au sein de cette approche. Ils divergent cependant sur la genèse du développement professionnel. Ainsi, pour certains auteurs (Nault, 1999; Vonk 1988; Zeichner et Gore, 1990), celui-ci débute en amont de la formation initiale (c'est la phase que les sociologues qualifient de socialisation pré professionnelle), alors que pour d'autres (Huberman, 1989), c'est l'entrée dans le métier qui en constitue l'amorce. En accord toutefois avec le fait que le développement professionnel constitue un continuum traversé par des phases, ces auteurs divergent également sur les limites initiales et finales de celles-ci. Face à une telle diversité, comment définir la notion de développement professionnel?

\section{Vers une définition du développement professionnel chez les enseignants}

"Que ce soit pour les postulants, les novices ou les chevronnés en enseignement, le développement professionnel se fait dans le sens de la modification des attitudes, des habiletés, des performances, des valeurs, de l'image de soi, du rapport au métier, de la perception à l'égard des élèves, des croyances, etc. " (Mukamurera, Uwamariya, 2005). Ainsi, « indépendamment des postures épistémologiques qui colorent les théories et les modèles (...), l'idée commune qui ressort est que le développement professionnel est un processus de changement, de transformation, par lequel les enseignants parviennent peu à peu à améliorer leur pratique, à maîtriser leur travail et à se sentir à l'aise dans leur pratique ». (Mukamurera, Uwamariya, 2005). C'est ainsi qu'il conviendra de comprendre cette notion lorsque nous l'emploierons.

Le dénominateur commun à ces définitions est/sont le(s) changement(s) : il apparaît comme l'élément central de la notion. Comment sont énoncés le(s) changement(s) qui s'opèrent au cours du développement professionnel ? Dans quelle(s) temporalité(s) sont-ils supposés s'inscrire? A quelle(s) condition(s) pense-t-on qu'ils peuvent-ils se réaliser? L'individu parvient-il à les rationaliser ? Peut-il les susciter, à défaut de les maîtriser ? Quels sont les outils institutionnels qui permettent de les appréhender? Ce sont les interrogations qui sous-tendent notre enquête empirique.

Voyons, dans un premier temps, quelles sont les étapes du développement professionnel enseignant identifiées par les textes officiels.

\section{La notion de développement professionnel à travers les textes officiels La construction de compétences, une dimension axiale du développement professionnel}

On constate que la notion de « développement professionnel » n'existe pas sur le portail «Eduscol » du ministère de l'éducation nationale. L'entrée dans le métier est intégrée au volet de la formation continue. Ainsi, les propos de Mukamurera et Uwamariya (2005), qui ont ouvert cet article, se vérifient. En effet, on constate bien une certaine confusion entre le sens de la notion elle-même et les moyens par lesquels le développement professionnel se réalise. 
Le développement professionnel : quels indicateurs?

On remarque aussi que la construction de compétences, individuelles et collectives, est au cœur du processus de développement professionnel: "La formation continue des enseignants vise à doter les professeurs des compétences professionnelles indispensables à une constante adaptation aux évolutions du système éducatif et à la réussite de tous les élèves. Elle concerne les enseignants du premier et du second degré ".

Cette dernière phrase mérite qu'on s'y attarde puisque le développement de compétences collectives est supposé entraîner, dans son sillon, des transformations identitaires (collectives) majeures qui sont censées s'appliquer, au-delà de leurs spécificités, à l'ensemble des enseignants du primaire et du secondaire. Loin de ne constituer qu'un idéal vers lequel tendre, ces transformations identitaires sont actées par le BO (Bulletin Officiel, numéro 1) de janvier 2007 qui institue un référentiel de compétences unique « pour tout type d'enseignant " au cahier des charges des IUFM : "La formation professionnelle initiale, dispensée en institut universitaire de formation des maîtres, doit permettre d'assurer une maîtrise suffisante de chacune des dix compétences suivantes (...). Au terme de l'ensemble des stages en responsabilité, le professeur des écoles stagiaire est supposé avoir construit des compétences professionnelles lui permettant d'envisager une prise de fonction efficace ».

\section{L'inscription du processus de développement professionnel dans un rapport au temps institutionnel}

D'un point de vue institutionnel, l'entrée dans le métier est considérée comme une période charnière du développement professionnel, depuis 2001, si l'on se réfère aux circulaires qui lui sont consacrées (circulaire du 27 juillet 2001 \& circulaire du 23 février 2007). L'expression "période décisive » de la carrière, mentionnée sur le portail Eduscol, confirme l'importance de cette phase.

Par ailleurs, la circulaire de 2007 permet de circonscrire, d'un point de vue institutionnel, une première phase du développement professionnel, celle au cours de laquelle, dans notre analyse, les néo-titulaires seront identifiés comme " primo débutants ". II apparaît en effet que les "enseignants débutants ", pour reprendre la nomenclature de l'éducation nationale, bien que titulaires, sont dorénavant considérés comme des enseignants en «formation initiale différée ", durant les deux premières années qui suivent la titularisation (c'est-à-dire T1 \& T2). De plus, les compétences des personnels enseignants, inscrites au référentiel, se construisent, progressivement, durant l'année de professionnalisation et "se développent et se consolident pendant les deux premières années d'exercice puis tout au long de la vie professionnelle » (BO $n^{\circ} 9$, 1er mars 2007). Ainsi, ces deux premières années constituent une étape, identifiable institutionnellement, d'un continuum au long cours. L'éducation nationale semble donc situer la genèse de ce continuum « en amont du concours et de la formation professionnelle en IUFM ", dès qu'un projet professionnel est formulé par le futur enseignant (BO n9, 1er mars 2007).

Quels sont les outils dont dispose l'institution pour permettre le développement des compétences des enseignants?

\section{Outils au service du développement professionnel, d'un point de vue institutionnel}

Au sein de ce continuum que nous venons de délimiter en amont et en aval, les stages de "pratique accompagnée » et ceux " en responsabilité » constituent institutionnellement l'élément central au service du développement professionnel. De la pratique va naître 
l'expérience qui, progressivement, au cours de la formation initiale, exigera d'être analysée au moyen d' "outils conceptuels et des apports de la recherche universitaire " (BO $n^{\circ} 9$, mars 2007) lors de la formation en IUFM. Ainsi, l'alternance, voire la dialogique, entre formation théorique et formation pratique, est officiellement au cœur de la formation initiale et continue.

Outre la réalité de l'alternance, c'est de "la qualité de la collaboration entre le lieu de stage et l'institut de formation » (BO n`9, mars 2007) qu'est censée dépendre la qualité de la formation et donc du développement professionnel. Ainsi, celui-ci dépendrait des expériences effectuées par les enseignants, des contextes éducationnels qu'ils découvrent mais également des collaborations et interactions avec leurs pairs, qu'ils soient collègues ou formateurs, et enfin d'eux-mêmes puisque «l'enseignant joue un rôle important dans son propre développement professionnel. II peut utiliser plusieurs moyens pour apprendre et maîtriser son métier, entre autre l'analyse de situations de la pratique, la participation à certaines activités, etc. Cela nécessite son intérêt personnel et son engagement » (Mukamurera, Uwamariya, 2005). Ces dimensions sont présentes dans BO $n^{\circ} 9$ (mars 2007). Les objectifs des stages en responsabilité s'y déclinent en effet sous forme de verbes d'action, tels: "participer (à l'ensemble des activités de l'établissement ou de l'école)", " agir », " rencontrer », " rechercher », qui sous-entendent l'engagement de l'acteur. Pour accomplir pleinement son engagement dans l'agir, le stagiaire est appelé à «mobiliser en situation professionnelle les savoirs construits à l'IUFM (formations disciplinaires et didactiques, analyses de pratique professionnelle) ".

Enfin, au terme de l'année de T2, la formation initiale s'achève et c'est par le biais de la formation continue, que l'enseignant, au-delà de sa pratique de terrain, continuera à cheminer dans ce continuum et à construire une identité professionnelle de plus en plus affirmée.

\section{Outils au service de l'évaluation du développement professionnel, d'un point de vue institutionnel}

En amont de la titularisation, ce sont les stages qui sont prétextes à l'évaluation. Parallèlement, un dossier de compétences « accompagne les professeurs durant l'année de professionnalisation et les deux premières années d'exercice (...). Ce dossier de compétences a une double fonction : il rend compte de la maîtrise des compétences attendues à la fin de l'année de stage et permet au jury de se prononcer dans le cadre de l'examen de qualification professionnelle; en attirant l'attention sur les compétences qui doivent être confortées, il facilite ensuite la construction du parcours de formation initiale différée sur les deux années qui suivent la titularisation » (BO n9 9 , mars 2007).

Ce découpage institutionnel du continuum fait-il sens pour les enquêtés?

\section{Le développement professionnel du point de vue des discours de professeurs des} écoles néo-titulaires

Les marqueurs temporels dans les discours, manifestations de l'amélioration des pratiques enseignantes

Notre corpus est constitué de trente entretiens semi-directifs, menés auprès de professeurs des écoles néo-titulaires, exerçant de la maternelle à la SEGPA (Sections d'Enseignement Général et Professionnel Adapté), dans les académies de Nancy-Metz et de 
Le développement professionnel : quels indicateurs?

Strasbourg. Pour le cadrage de l'étude, nous nous sommes appuyés sur l'enquête menée par Pierre Périer (2003). Selon le chercheur, les enseignants manifesteraient une centration sur les savoirs disciplinaires et leur transmission à mesure qu'ils avancent dans la carrière, les premiers changements notoires devenant visibles après quatre ou cinq années d'ancienneté. Par conséquent, les néo-titulaires sont, pour nous, des professeurs des écoles ayant d'une à trois années d'expérience sur le terrain. L'Education nationale les nomme T1, T2 ou T3 (T comme " titulaires "), répartis ainsi : 18 T1, 5 T2 et 7 T1, dont 11 hommes et 19 femmes. Les travaux de Périer permettent donc non seulement d'identifier les néo-titulaires mais également de procéder à un premier découpage au sein du continuum. Nous tenterons précisément de comprendre le processus de développement professionnel à partir de cette phase, que nous dénommerons « entrée dans le métier ».

Nous avons, pour cela, dans un premier temps, procédé à un relevé systématique des marqueurs temporels présents dans les entretiens intégralement retranscrits. Dans un second temps, nous avons isolé et retenu ceux auxquels était adjointe une expression contenant l'idée de progrès, matérialisée le plus fréquemment par un verbe exprimant le constat d'un changement positif. Enfin, nous avons procédé à un regroupement thématique de l'ensemble des éléments retenus et associés à l'idée de changement. Trois axes heuristiques, que nous serons amenés à développer, ont émergé: le développement professionnel s'accompagne d'une évolution du rapport à soi, aux autres (en l'occurrence aux élèves) et au métier.

Par ailleurs, pour permettre le dialogue entre les textes officiels et les discours des enquêtés, nous avons exploré le rapport à la formation initiale et continue des interviewés. Dans la même optique, nous avons voulu identifier les acteurs du système et les outils qu'ils considèrent comme des adjuvants à leur développement professionnel. Nous avons également tenté de repérer et de mettre au jour, à l'aide du relevé des indicateurs temporels, les étapes constitutives du développement professionnel pour ces enseignants, depuis leur première rentrée jusqu'au moment de l'entretien, à partir du repérage des points de convergence susceptibles de s'établir au fil du temps. Ces étapes sont-elles invariablement les mêmes pour tout enseignant ? Dans quel rapport au temps les enseignants néo-titulaires vivent-ils leur développement professionnel ?

\section{L'inscription du processus de développement professionnel dans un rapport au temps personnel}

A y regarder de plus près, l'expression « rapport au temps ", employée au singulier, s'avère inexacte. Le processus de développement professionnel s'ancre manifestement dans une dualité de rapports au temps : le rapport au temps que vit le sujet et le rapport au temps dans lequel l'institution l'inscrit. Le temps institutionnel ayant été mis précédemment au jour, nous nous attacherons, dès à présent, à mettre en exergue le rapport personnel au temps qui émerge des entretiens menés avec les professeurs des écoles néo-titulaires.

Ce sont les sentiments d'auto-efficacité et de progrès qui permettent au sujet le passage, symbolique, entre les unités temporelles qui ponctuent l'entrée dans le métier. Si la durée et le nombre de phases diffèrent d'un individu à l'autre, il semblerait néanmoins qu'une phase soit identique pour tout néo-titulaire: celle au cours de laquelle celui-ci se sent «primo débutant ». 


\section{$T 1$ ou l'ère des "primo débutants"}

Cette phase se caractérise par un rapport subi au temps : c'est l'ère de l'urgence, de l'extensivité du temps professionnel sur le temps social et d'un certain nombre de dysfonctionnements dans la gestion de la classe. Le « terrain ", par opposition à la formation, jugée globalement trop théorique, se révèle un lieu propice à un apprentissage rapide du métier. Le recueil des marqueurs temporels montre que la phase au cours de laquelle l'enseignant se perçoit "primo débutant ", variable d'un sujet à l'autre, peut être très brève. C'est l'idée résumée par les propos d'Emmanuelle (T1) qui témoignent d'une progression rapide, associée à un sentiment d'amélioration de la gestion de classe et de la relation à l'élève :

« J'ai vite compris quand j'ai réussi à voir les différences de niveau et à adapter, eh bien que l'élève était suffisamment rassuré pour qu'après les choses du ressort du comportement puissent en fait s'améliorer, quoi, j'arrivais après à mettre en place un autre travail ».

D'étapes en étapes, de réussites en réussites, signifiées également par des verbes porteurs de l'idée d'une amélioration (tels ici réussir, comprendre, arriver), elle quitte ce statut de "primo débutante", que traduit l'expression "au début" et qui débouche sur un "après". Si les indicateurs temporels, tels ceux utilisés par Emmanuelle, sont flous et ne permettent pas de cerner précisément la période des «tout débuts ", d'autres révèlent que des améliorations sont palpables " au bout de quelques mois ", plus précisément " au bout de six mois". Pour d'autres enquêtés, la fin de la première période constitue une étape charnière, elle se situe environ deux mois après la rentrée scolaire.

Au final, nous pouvons dire que la durée de cette période est fluctuante et singulière : elle peut s'échelonner sur plusieurs semaines, voire plusieurs mois. Quoi qu'il en soit, des changements significatifs, semblent se cristalliser, globalement, au bout de quelques mois et, de manière certaine, au terme de la première année scolaire. L'institution serait-elle consciente de cette rapide progression qui se joue à l'orée de la carrière pour la formaliser, par le passage d'un échelon, au bout de trois mois d'exercice?

Quels sont les changements concrets, précédemment évoqués, qui accompagnent le sentiment de progression exprimé par les enquêtés?

\section{Nature des transformations associées au sentiment de développement professionnel}

L'analyse des discours révèle que, de manière globale, l'expérience de terrain est source d'apprentissages, non nécessairement définis de manière précise par les enquêtés, comme en témoignent des formulations telles : "J'ai appris beaucoup de choses sur le terrain ".

Lorsque la nature des apprentissages est définie, on constate que l'immersion dans le terrain permet aux enseignants de modifier leurs conceptions initiales du métier ou, du moins, de déconstruire celles qui sont erronées:

« J'avais un mi-temps et deux quarts temps. Et je me suis dit que le mi-temps se passerait très bien mais que les quart-temps, (...) ce serait plus difficile (...). C'était vraiment une relation très forte alors que, comme dit, c'est allé à l'encontre de ce que je pensais en début d'année » (Yann, T2) ;

« II y avait un gros décalage entre ce que j'imaginais et ce que c'était réellement. (...) je suis arrivée avec des illusions, vraiment. J'avais l'impression 
Le développement professionnel : quels indicateurs?

que ça allait être magique : un peu la classe au pays de Barbie » (Claire, T2, à propos du premier stage en responsabilité).

Si Yann est, par ailleurs, pleinement conscient de sa progression (« J'ai quand même beaucoup évolué depuis [la première rentrée] "), des mécanismes semblent également travailler, à son insu, puisqu'il a parfois du mal à expliquer les causes des transformations positives qu'il vit dans sa classe. Ainsi, face à la conseillère pédagogique qui l'incite à analyser l'amélioration de sa pratique constatée, celui-ci répond:

"C'est la question que je me pose moi-même. J'ai du mal à répondre à cette question-là. J'ai peut-être fait des erreurs. C'est difficile d'analyser son propre comportement » (Yann, T2).

On peut constater que les progrès des élèves constituent, pour lui, un bon indicateur de sa propre progression :

"Et au bout d'un mois, un mois et demi, quand les choses sont rentrées dans l'ordre et que j'ai vu que les élèves commençaient à progresser, ça m'a vraiment donné envie de continuer. (...) le fait de voir que je suis arrivé à quelque chose avec les élèves me donne envie de continuer. Est-ce que c'est allé mieux pour moi parce que j'ai vu que les gamins progressaient et donc, ça m'a donné plus envie et plus confiance ou est-ce que les gamins..., ou l'inverse ? Je sais pas. Mais ce qui est sûr, c'est que c'est lié " (Yann, T2).

II ressort des propos de Yann que le sentiment de progression, d'auto-efficacité est source de motivation.

Au-delà des répercussions purement professionnelles qui accompagnent le processus de développement personnel, des modifications du rapport à soi sont tangibles. Ainsi, Yann se dit "plus confiant" et déclare avoir "abordé de manière beaucoup plus sereine " la deuxième rentrée. Meryem (T3) parvient, quant à elle, à faire le distinguo entre soi personnel et soi professionnel, dans sa pratique. Le développement professionnel s'est donc accompagné d'un processus de distanciation : "au début, les échecs, enfin..., l'échec du gamin, on le prend pour soi, en fait ».

L'expérience permet aux enseignants qui adoptent une posture réflexive, de modifier leurs pratiques et notamment les situations d'apprentissage qu'ils proposent à leurs élèves : " J'avais commencé par un projet sur la littérature migrante. Ça a été une tentative d'approche. II est évident que si je devais présenter ce projet maintenant, (...) c'est clair que mes objectifs, il faut que je les revoie parce que c'était à moitié adapté (...). Mais, hum, voilà, ce que je pensais à l'époque " (Emmanuelle, T1).

Enfin, c'est en termes de gestion de classe que les progrès sont palpables : "On va dire que le bilan, c'est que j'ai beaucoup appris en terme de gestion de classe» (Paul, T1).

L'apprentissage du métier : un continuum au service du développement professionnel

Si l'ensemble des enquêtés paraît conscient que l'apprentissage du métier se construit progressivement, avec les adjuvants majeurs que sont le temps, l'expérience et la posture réflexive, les femmes verbalisent davantage que leurs homologues masculins sa dimension toujours inachevée, c'est-à-dire " tout au long de la carrière ", pour citer Emmanuelle (T1). Elles mettent plus facilement en avant la dimension toujours perfectible des pratiques, 
comme en témoigne la réitération des modalisations temporelles « toujours " et les dérivés, en italiques, construits autour du noyau « mieux ». II s'agit, selon Zoé (T3), en effet de :

"Euh..., toujours évoluer pour faire en sorte que, de tirer, pas des leçons, mais améliorer, euh..., son enseignement, toujours remettre en question ce qu'on a fait pour voir où le..., la chose pourrait être améliorée pour faire mieux, à chaque fois. Non, en toute séance, il y a toujours des imperfections et euh..., il y a des séances qui se passent très bien, d'autres qui se passent moins bien. Faut savoir les analyser, savoir ce qu'on peut mettre en place pour les améliorer » (Zoé, T3).

Cette dernière considère la dimension perpétuellement inachevée, qui caractérise le continuum, comme une difficulté majeure liée à l'exercice du métier puisque c'est au moment où sont abordées, pendant l'entretien, les difficultés du métier, qu'elle met en avant l'infinie et l'atemporelle perfectibilité de la pratique professionnelle.

Peut-on trouver ici des indicateurs d'une éventuelle différenciation sexuée du rapport au développement professionnel? Cette piste demanderait à être travaillée.

A partir de l'année de T3, le dispositif de formation continue n'est plus qu'alimenté par des stages, non obligatoires, inscrits au plan de formation, et par dix-huit heures annuelles d'animations pédagogiques obligatoires. Comment les enquêtés T3 perçoivent-ils ce dispositif ? On distingue deux groupes de T3. II y a, d'une part, ceux qui déplorent le manque de formation continue, à l'image de Meryem ou d'Annabelle :

"La formation continue, elle devrait être..., elle devrait être importante en début d'expérience parce que là, on est vraiment tous les jours confronté à des difficultés et puis, ouais... " (Meryem, T3) ;

"Au cours de l'année, ben, on a eu deux animations pédagogiques chacune de deux heures : autant dire que c'est assez léger » (Annabelle, T3).

Ces enseignantes, conscientes de leurs progrès, reconnaissent le côté perfectible de l'apprentissage du métier, mis en valeur par l'expression réitérée " pas encore » :

" (...) souvent, je ressens quand même le manque d'expérience parce que les enfants arrivent avec des difficultés, alors, même si j'arrive à les relever, les difficultés, après, les analyser et pouvoir y remédier, c'est encore différent » ou encore « Je ne me sens pas encore assez prête », " je ne me sens pas encore assez solide ", " je ne me vois pas encore... " (Annabelle, T3).

Au regard d'une formation initiale vivement critiquée et d'une formation continue quasi inexistante, elle a décidé de poursuivre parallèlement une formation en sciences de l'éducation à l'université :

« Je pourrais très bien passer le CAPA-SH et j'ai pas envie de me former chez eux. Je..., je n'ai pas confiance, en fait. Et c'est pour ça que j'ai repris des études, pour me former à l'extérieur ".

II y a, d'autre part, un second groupe de T3 qui émerge. Ce groupe se distingue par l'absence d'évocation d'un quelconque besoin de formation continue. Doit-on en conclure que ceux-ci se sentent suffisamment armés pour ne pas évoquer ce manque de formation, évoqué par l'autre groupe de T3 ? C'est ce que conduisent à penser les propos de Karine (T3) qui conjugue au passé les difficultés: "C'était difficile au début. C'était beaucoup de travail ». Ou, au contraire, ces discours aseptisés de difficultés traduisent-ils la crainte de 
Le développement professionnel : quels indicateurs?

passer pour un apprenti aux yeux de l'enquêteur alors qu'on est T3, titularisé et inspecté, c'est-à-dire reconnu comme n'étant plus en «formation initiale intégrée »? Par ailleurs, on pourrait s'interroger, d'un point de vue épistémologique, sur le biais éventuel que peut constituer la situation d'entretien d'elle-même : l'enquêtée, en l'occurrence ici Karine, prise dans ce rapport asymétrique d'enquêteur à enquêté, déploierait une stratégie ayant pour visée de plaire à l'enquêteur et de se montrer sous les meilleurs hospices possibles.

\section{Aides et outils personnels au service du développement professionnel}

En formation initiale, ce sont les stages qui sont estimés, par l'ensemble des enquêtés, comme l'élément le plus formateur, quand bien même ils sont globalement jugés trop peu nombreux. Ils permettent notamment de déconstruire les représentations initiales erronées et de limiter, sans doute, les écarts entre la vision idéalisée et imaginaire du métier et la réalité. Ainsi, Paul (T1) déclare n'avoir point trop appréhendé sa première rentrée en ZEP pour avoir eu « des représentations initiales qui étaient pas trop, voire pas du tout décalées par rapport à la réalité du terrain, en fait, vu ce que l'IUFM m'avait permis de voir en allant dans des classes de ZEP, l'année d'avant ».

L'écrit professionnel, dont les modalités diffèrent d'un IUFM à l'autre, devrait être un outil au service de l'analyse des pratiques pour des enseignants que l'institution souhaite voir devenir des praticiens réflexifs, au sens de Schön. Mais l'usage évaluatif que fait l'IUFM de cet écrit ne dénature-t-il pas les fonctions formatives et réflexives pour lesquelles il était pensé ?

Une fois titularisés, sur le plan des ressources humaines, les collègues sont ceux qui sont le plus fréquemment cités par les enquêtés, notamment dans le cadre des postes à mi-temps lorsque la coopération est heureuse. Ils ont pour dénominateur commun le fait d'être perçus comme des acteurs expérimentés, ayant permis, aux débutants, une amélioration des pratiques, par les conseils qu'ils dispensent et leur écoute bienveillante, notamment :

"J'ai appris beaucoup de choses, au niveau des collègues, qui m'ont beaucoup aidée, vraiment beaucoup apporté de..., de pratiques, de savoirfaire, de connaissances..., pour bien mener la classe » (Zoé, T3).

Sur le terrain, l'analyse de pratiques devient donc informelle puisqu'elle se "désinstitutionnalise" progressivement à la sortie de l'IUFM. Aussi, pour combler ce manque, certains enquêtés, T1, ont pris l'initiative de se retrouver entre débutants, à l'IUFM, de manière hebdomadaire afin de promouvoir les échanges entre pairs, le travail collaboratif et l'analyse, informelle, de pratiques. On peut supposer aussi que le choix de se retrouver à I'IUFM n'est pas neutre : il permet sans doute un accès facilité à la documentation, ressource au service du développement professionnel. Ces groupes se constituent sur le modèle des communautés de pratique.

Les conseillers pédagogiques, quant à eux, n'apparaissent pas de manière évidente, dans les discours, comme des aides, au service du développement professionnel. Leur proximité avec les services d'inspection conduit certains enquêtés à les percevoir davantage comme des « mini inspecteurs » (Annabelle, T3) que comme des conseillers à part entière.

Enfin, le "terrain » occupe une position prévalente. II est en effet perçu, dans les discours, comme un cadre au sein duquel se construisent les pratiques, perfectibles, et comme un moyen puisqu'il est le terreau favorable à l'augmentation du capital expérience. Les enseignants que nous avons interrogés disent donc apprendre par et sur le terrain. La 
formation dispensée par l'IUFM n'apparaît plus que comme un arrière-plan, dans laquelle certains vont aller ponctuellement puiser alors que d'autres, à l'image de Paul (T1), vont faire le choix de faire fi de certaines méthodologies conseillées par la formation pour construire les situations d'apprentissage, et que d'autres encore, telle Astrid (T3), entament un " désapprentissage » de ce qu'ils ont appris à l'IUFM.

\section{Discussion}

\section{Temps de la formation, temps de l'évaluation : quelles relations ?}

Les modifications institutionnelles récentes, liées à l'accompagnement dans l'entrée dans le métier et entérinées par la circulaire du 23 février 2007, signifient que cette phase est reconnue, par le ministère, comme une phase charnière du développement professionnel enseignant. Aussi, l'introduction du dispositif de "formation initiale intégrée ", dans le plan de formation des T1 et T2, vient-elle conforter l'idée que cette phase est décisive pour la carrière de l'enseignant débutant. Sur ce point, les textes officiels convergent avec l'opinion des enquêtés qui valorisent davantage cette formation, "en circonscription ", que la formation initiale. De plus, l'introduction de ce nouveau dispositif dans la formation professionnelle véhicule implicitement l'idée que le développement professionnel se construit progressivement. Or, c'est au cours de l'année de T2 que les « titulaires en formation initiale intégrée » - expression oxymoresque - "subissent » leur première inspection. Si le temps de l'évaluation et celui de la formation se chevauchent, le temps de la formation peut être dénaturé. On peut en juger par l'exemple de Brigitte, T1, qui a vécu une inspection pendant son temps de formation, cette situation génère de l'angoisse par rapport aux futures inspections, ébranle la confiance en soi et aurait pu conduire à la démission :

"Donc, moi, mon inspection, l'année prochaine, je vais être mais dans mes petits souliers... Ça m'a..., j'en ai été malade, quoi, mais vraiment ! Si j'm'étais pas dit euh, en moi même que: "si, si, j'ai envie d'le faire, et puis que j'me sentais bien, et que j'avais..." ".

Ces deux temporalités, que sous-tendent des logiques différentes, ne devraient-elles pas être clairement distinguées pour que leur valeur en reste préservée?

\section{Outils de formation, outils d'évaluation}

Dans le même registre, le statut de l'écrit professionnel est également ambivalen, voire bafoué. II est en effet à la fois un instrument au service des apprentissages, qui doit permettre réflexion au service, in fine, de l'acquisition de compétences nouvelles, et support d'évaluation sommative. Le stagiaire osera-t-il librement exprimer ses difficultés ou, au contraire, concevra-t-il cet exercice exclusivement comme une tâche à laquelle il lui faudra se plier de manière scolaire en la conformant aux critères d'évaluation?

\section{Une formation " dévalorisée "}

Si la formation est parfois discréditée par les stagiaires parce qu'elle ne répond pas à leurs attentes, on peut également constater que l'institution elle-même semble travailler à sa dévalorisation. Elle n'hésite pas, en effet, à placer des débutants sur des postes à profils qui exigent, théoriquement, la détention d'un diplôme spécialisé (de type CAPA-SH ou une certification en langue) ou encore à "parachuter dans le vide » (Karine, T3) des personnes 
Le développement professionnel : quels indicateurs?

de liste complémentaire. Ces solutions temporaires ne véhiculent-elles pas, en filigrane, le raccourci qu'un apprentissage sur le tas est possible, voire suffisant?

\section{Discours institutionnels, discours personnels}

En termes d'accompagnement à l'entrée dans le métier, il semblerait qu'il y ait un écart entre les idéaux des politiques éducatives et la réalité perçue par les enseignants, sur le terrain. Ce constat est convergent avec celui effectué par Marcel (2005) : "Les différentes modalités de la prescription [conséquente à la loi d'orientation de 1989] ne rencontraient pas un écho uniforme sur le plan des pratiques enseignantes". La notion même d'accompagnement, au cœur de la circulaire de 2007, est en effet absente des discours personnels. Les néo-titulaires n'hésitent pas à se dire "largués " (Claire, T2), sur le terrain, dès le premier stage. Lilian déplore d'ailleurs le fait que les stages de pratique accompagnée soient trop peu nombreux au cours de la formation initiale. Aussi déclare-t-il avoir apprécié celui dont il a pu bénéficier en $\mathrm{T} 1$.

On constate également que, si des étapes du développement professionnel ont pu être mises au jour dans les discours personnels et officiels, le découpage de celles-ci est complexe puisque des variations interpersonnelles existent, au-delà des points de convergence, d'un professionnel à l'autre. Ceci justifierait peut-être un accompagnement plus individualisé dans l'entrée dans le métier mais des freins économiques s'y heurteraient sans doute.

Enfin, si les néo-titulaires adhèrent au dessein de développement d'analyse des pratiques, souhaité par les politiques éducatives, ils ne se sentent néanmoins pas armés des " outils conceptuels " que prône pourtant l'institution.

Gageons que c'est peut-être parce que la nouvelle politique d'accompagnement à l'entrée dans le métier est sans doute encore trop récente que son effectivité est discutée.

\section{Conclusion}

Notre recherche a permis de mettre au jour la présence d'indicateurs du développement professionnel dans les discours officiels et dans ceux produits par les enseignants sur l'entrée dans le métier.

A linterface des deux corpus, on trouve les indicateurs temporels. Ils se déclinent en indicateurs (temporels) personnels et en indicateurs (temporels) institutionnels.

Les indicateurs personnels sont des indicateurs d'auto-évaluation du développement professionnel. Elaborés de manière informelle par les enseignants, ils permettent une traçabilité, dans le temps, du sentiment de progression ou d'auto-efficacité, lié à l'acquisition de compétences ou à l'évolution des représentations. On constate des variations interpersonnelles dans la production de ces indicateurs, inscrivant leurs auteurs dans une pluralité de rapports au temps. A contrario, le temps institutionnel s'écoule selon un découpage identique pour chaque enseignant. L'inscription de l'enseignant dans ce double rapport au temps ne constitue-t-elle pas un frein à la qualité de son développement professionnel? 


\section{Bibliographie}

Bru, M. (2004). Quelles compétences pour enseigner ? In V. Hajjar \& A Baubion-Broye, (Ed.). Modèles et méthodologies d'analyse des compétences : le double éclairage des pratiques et des recherches. Toulouse : Octares.

Bru, M. \& Talbot, L. (2005). Des compétences pour enseigner, entre objets sociaux et objets de recherche. Paris : PUF.

Borko, H. (2004). Professional development and teacher learning: Mapping the terrain. Educational Researcher, 33(8), 3-15.

Boucher, L.P. \& L'Hostie, M. (1997). Le développement professionnel continu en éducation. Nouvelles pratiques. Québec : Presses de l'Université du Québec.

Day, C. (1999). Developing teachers. The challenge of lifelong learning. Londres : Palmer Press.

Clement, M. \& Vandenberghe, R. (1999). Teachers' professional development : A solitary or collegial (ad)venture ? Teaching and Teacher Education, 16, 81-101.

Condorcet, J.-A. (1792). Rapport sur l'organisation générale de l'instruction publique. Paris.

Dolan, S.-L., Lamoureux, G. \& Gosselin, E. (1995). Psychologie du travail et des organisations. Montréal : Gaëtan Morin.

Elmore, R.F. (2002). Bridging the gap between standards and achievement : The imperative for professional development in education. Washington: Albert Shanker Institute.

Garet, M.S., Porter, A.C., Desimone, L., Birman, B.F. \& Suk Yoon, K. (2001). What makes professional development effective? Results from a national sample of teachers. American Educational Research Journal, 38(4), 915-945.

Glatthorn, A. (1995). Teacher development. In W. Anderson (Ed.), International encyclopaedia of teaching and teacher education, 41-46. Cambridge : Cambridge University Press.

Gouvernement du Québec (1999). Orientations pour la formation continue du personnel enseignant. Québec : Ministère de l'Éducation du Québec.

Guskey, T.R. (2002). Does it make a difference ? Evaluating professional development. Educational Leadership, 59(6), 45-51.

Huberman, M. (1989). Les phases de la carrière enseignante : un essai de description et de prévision. Revue française de pédagogie, 80, 5-16.

Huberman, M., Grounauer, M. \& Marti, J. (1989). La vie des enseignants : évolution et bilan d'une profession. Neuchâtel-Paris : Delachaux/Niestlé.

Killeavy, M. (Ed.). (2001). Professional development in teacher education: Some international perspectives. European Journal of Teacher Education, 24(2), 1-251.

Lafortune, L., Deaudelin, C., Doudin, P.-A. \& Martin, D. (Ed.). (2001). La formation continue. De la formation à la réflexion. Québec : Presses de l'Université du Québec.

Marcel, J.-F. (2005). Le développement professionnel au travers de l'évolution des pratiques enseignantes. Revue des sciences de l'éducation, 31 (3), 585-606.

Marcel, J.-F. (2007). L'expérience professionnelle de l'enseignant du primaire In M. Bru \& L. Talbot (Ed.). Des compétences pour enseigner. Entre objets sociaux et objets de recherche. Rennes : Presses Universitaires de Rennes.

Marcel, J.-F (2007). Nouvelles pratiques enseignantes et développement professionnel in J.-F. Marcel, V. Dupriez, V., D. Perisset Bagnoud et M. Tardif (Ed.). (2007). Coordonner, collaborer, coopérer : de nouvelles pratiques enseignantes. Bruxelles : De Boeck. 
Le développement professionnel : quels indicateurs?

MEN (2001). Circulaire relative à l'accompagnement de l'entrée dans le métier et à la formation continue des enseignants des premier et second degrés et des personnels d'éducation et d'orientation. Paris : Education Nationale.

MEN. (2007). $B O n^{\circ} 1.4$ janvier \& $n^{\circ} 9.1^{\text {er }}$ mars.

MEN. Site Eduscol. [http://eduscol.education.fr] [consulté le 10.03.2009].

Nault, T. (1999). Les forces d'incubation pour un moi professionnel personnalisé en enseignement. In J.-C. Hétu, M. Lavoie \& S. Baillauquès (Ed.), Jeunes enseignants et insertion professionnelle. Bruxelles : De Boeck, 139-159.

Périer, P. (2003). Le métier d'enseignant dans les collèges et les lycées au début des années 2000. Les dossiers d'éducation et formation, 145.

Uwamariya, A., Mukamurera, J. (2005). Le concept de « développement professionnel » en enseignement : approches théoriques. Revue des sciences de l'éducation, 31 (1), 133-155.

Vonk, J.-C. (1988). L'évolution professionnelle des enseignants débutants et ses répercussions sur la formation initiale et continue. Recherche et formation, 3(3), 47-60.

Wilson, S.M., Berne, J. (1999). Teacher learning and the acquisition of professional knowledge : An examination of research on contemporary professional development. In A. Iran-Nejad, P.D. Pearson (Ed.). Review of research in education, 24 (1), 173-209.

Zeichner, K. M., Gore, J. (1990). Teacher socialization. In R. W. Houston (Ed.). Handbook of research on teacher education. New York: Macmillan, 329-348 